\title{
Lemon juice counteracts the effect of green tea decoction on body weight gains, high fat diet Induced-liver steatosis, Total antioxidant status and some metabolic parameters in rats
}

\author{
Zakia Ben Abid $^{1}$, Abdelmajid Trimeche ${ }^{2}$, Houda Abaidi ${ }^{1}$, Sabri Denden ${ }^{1}$, Sami Fattouch ${ }^{3}$, \\ Mohamed Habib Jaafoura ${ }^{4}$, Mohamed Hédi Hamdaoui ${ }^{1}$ \\ ${ }^{1}$ Research Unit of Antioxidant Compounds, Oxidative Stress, Trace elements and Metabolic diseases, School of Health Sciences and \\ Technical of Tunis, University of Tunis El Manar, Tunis, Tunisia \\ ${ }^{2}$ National Institute of Nutrition, Tunis, Tunisia \\ ${ }^{3}$ LIP-MB Laboratory, INSAT, Tunis, Tunisia \\ ${ }^{4}$ Faculty of Medicine of Tunis, Tunis, Tunisia

\section{Email address:} \\ hamdaouimeh@gmail.com (Z. B. Abid), abdelmajid.trimeche@topnet.tn (A. trimeche), h.abaidi@yahoo.fr (H. Abaidi), \\ denden_sabri@yahoo.fr (S. Denden), sami.fattouch@insat.rnu.tn (S. Fattouch), siteweb.fmt@gmail.com (M. H. Jaafoura), \\ mh.hamdaoui@esstst.rnu.tn (M. H. Hamdaoui)
}

\section{To cite this article:}

Zakia Ben Abid, Abdelmajid Trimeche, Houda Abaidi, Sabri Denden, Sami Fattouch, Mohamed Habib Jaafoura, Mohamed Hédi Hamdaoui. Lemon Juice Counteracts the Effect of Green Tea Decoction on Body Weight Gains, High Fat Diet Induced-Liver Steatosis, Total Antioxidant Status and Some Metabolic Parameters in Rats. International Journal of Nutrition and Food Sciences.

Vol. 4, No. 1, 2015, pp. 1-7. doi: 10.11648/j.ijnfs.20150401.11

\begin{abstract}
Background: In previous studies, we reported that green tea decoction (GTD) cooked in boiling water for 15-min (15-min GTD) reduced body weight gain (BWG), glucose and lipid absorption and improved total antioxidant status (TAS) of rats fed normal or high-fat diet (HFD). However, the long-term effect of a mixture: 15-min GTD + lemon juice (LJ) on BWG, liver steatosis (LS), TAS, blood glucose and lipids is still unknown. Purpose The aim of the present study is to examine the long-term effect of 15-min GTD + LJ mixture on above parameters in rats fed HFD induced-obesity and LS. Methods Twentyeight male rats were assigned to four groups with comparable body weights. During 9-weeks treatment, rats were given adlibitum a basic diet (BD) or HFD to promote obesity and LS. The group 1 received the BD (BD group), the group 2 received HFD (HFD group), the group 3 received HFD + 15-min GTD (GTD group) and the group 4 received HFD + a mixture: 15-min GTD and LJ (GTDLJ group). Results The rats fed HFD had the highest BWG associated with an abundant LS spread than rats consumed BD. However, 15-min GTD significantly reduced the BWG and LS spread $(\mathrm{p}<0.001)$. GTD group showed also decreased plasma glucose, triglycerides and increased both TAS and GPX activities ( $p<0.05$ and $\mathrm{p}<0.01$, respectively). Surprisingly, an abundant reappearance of several LS spread and a restore of BWG were observed in GTDLJ group compared to GTD group. The GTDLJ mixture also reversed the GTD effect on plasma glucose, TAS, GPX and blood iron. Conclusion These results show that 15-min GTD significantly reduces BWG, HFD induced-LS, plasma glucose, triglycerides, blood iron and improves TAS and GPX. However, the GTDLJ mixture partially or totally counteracts the effect of 15-min GTD on BWG, LS, plasma glucose, blood iron, TAS and GPX.
\end{abstract}

Keywords: HFD Induced-LS, 15-Min GTD, GTDLJ Mixture, Body Weight Gains, Antioxidant Process

\section{Introduction}

The obesity state, excess of hepatic lipids accumulation and oxidative stress contribute to the development of nonalcoholic fatty liver diseases, in particular, liver steatosis
(LS) $[1,2]$. The LS increases the extent of cellular injury and liver lipids by enhancing lipogenesis and impairs of lipid transport. It also alters other liver tissue functions such as liver marker enzymes including ALT and AST activities [3, 4]. Other studies have observed a dramatic extent of 
oxidative stress because of the hepatic antioxidant defenses decline in liver steatosis cells [5].

Epidemiological evidence and randomized controlled studies have reported an inverse relationship between habitual tea consumption and body fat levels [6]. Therefore, more attention was focused on tea consumption because both green and black teas contain numerous polyphenolic compounds and caffeine that are able to better control the overweight gain and its complications [7]. Nevertheless, the most of study investigations on tea-polyphenol have explored tea leaves, green, oolong and black tea extracts or their derivatives (catechins, theaflavins) but not green tea decoction (GTD) which is a popular beverage through large areas of North Africa countries, especially in Tunisia. GTD is different from the green tea infusion (GTI), which considered as the usual beverage consumed in most of western countries. GTI is prepared by adding a portion of dried green tea leaves to about $1,000 \mathrm{ml}$ of hot water and let them brewed for about 3-min. However, the GTD is prepared by cooking the dried tea leaves in boiling water for a variable period of time, overall not exceeding $60 \mathrm{~min}$. Since, the highest level of polyphenolic compounds released in GTD is obtained after cooking the dried tea leaves for 15min as compared to GTD cooked for $30,60 \mathrm{~min}$ or to GTI [8]. Recent investigations showed that 15-min GTD decreases glucose absorption, improves glucose tolerance and antioxidant status and exert a potent antimicrobial effect as compared to those of 30- or 60-min GTD or GTI [8, 9]. It has been shown that the beneficial effect of 15-min GTD mainly depends to the preservation of its bioactive polyphenolic compounds after the cooking process.

On the other hand, in some area, tea is commonly consumed with milk, honey or citron lemon because of their delicious taste. GTI flavored with a slice of lemon is one of the most popular drinks, especially in western countries. However, lemon juice ( $\mathrm{LJ})$ is known for its high ascorbic acid (AA) content $(-40-50 \% \mathrm{w} / \mathrm{w})$ which could interact with tea-polyphenols and likely overcome its beneficial effects [10]. In a previous study, we reported that the addition of low doses of AA to GTD was able to overcome the inhibiting effect of GTD on iron absorption and improved its bioavailability from a typical Tunisian meal fed to rats [10]. However, the effect of the interaction between GTD and LJ on body weight gains, HFD induced-LS, antioxidant status and some related metabolic parameters is still unknown. Therefore, the study of the synergistic or antagonist effect that may occur between GTD-polyphenols and LJ has a great practical importance in human and animal nutrition.

The objective of this study is to examine the long-term effect of 15-min GTD alone or combined to fresh LJ given to rats fed HFD on body weight gain, HFD induced-LS, total antioxidant status (TAS) and some related metabolic parameters.

\section{Materials and Methods}

Preparation of the 15-min GTD and the mixture: green tea decoction-lemon juice (GTDLJ)

The 15-min GTD was freshly prepared throughout the experimental feeding period (EFP). An amount of $100 \mathrm{~g}$ of green tea leaves (Camellia sinensis L.) was purchased from local market in Tunis city and was boiled in 1,000 $\mathrm{ml}$ of distilled water for $15-\mathrm{min}$, and then cooled to room temperature before distribution. Moreover, a peeled fruit of citrus lemon (Citrus Limon, L.) was pressed in a robot and filtered to acquire a freshly LJ. The GTDLJ mixture was obtained by adding a volume of $200 \mathrm{ml}$ of LJ to $1,000 \mathrm{ml}$ 15-min GTD and mixed. During EFP, each rat consumed an average of $24 \mathrm{ml}$ of GTDLJ mixture/day/ which correspond to $4 \mathrm{ml}$ of LJ. Based on this volume and considering that $\mathrm{LJ}$ contained between 40 to $50 \% \mathrm{AA}$, we can deduce that GTDLJ mixture could provide roughly $2 \mathrm{mg}$ AA to rat/daily. This amount of AA is adequate for rats because this animal did not need high AA requirement as compared to human who needs about $70 \mathrm{mg} /$ daily.

\subsection{Diets}

The basic diet (BD) and high fat diet (HFD) given to rats during the EFP are presented in table 1.

Table 1. Composition of basic (BD) and high-fat diet (HFD), $\mathrm{g}_{\mathrm{kg}}^{-1}$ diet

\begin{tabular}{lll}
\hline Ingredients & BD & HFD \\
\hline Powder skim milk $^{1}$ & 400 & 400 \\
Vegetable oil $^{\text {Mutton fat }}{ }^{2}$ & 50 & - \\
Animal butter $^{\text {Cholesterol }}$ & - & 200 \\
Maize starch $_{\text {Sucrose }}^{3}$ & - & 100 \\
$\mathrm{CaCO}_{3}$ & 390 & 10 \\
$\mathrm{Na}_{3} \mathrm{PO}_{4}$ & 100 & 200 \\
$\mathrm{NaCl}_{\mathrm{KCl}}$ & 20 & 30 \\
$\mathrm{Vitamin}$ & 20 & 20 \\
$\mathrm{Mineral} \mathrm{mixture}^{5}$ & 5 & 20 \\
\hline
\end{tabular}

${ }^{1}$ As source of protein (Inesfood- Tunisia). ${ }^{2}$ From subcutaneous adipose tissue of mutton born and breeding in Tunisia. ${ }^{3}$ Cholesterol Codex (Chemipharma-Tunisia).

${ }^{4}$ Vitamin mixture (per kilogram dry weight of diet): synthetic vitamin A concentrate $=6.500 \mathrm{IU}$; cholecalciferol $=1.300 \mathrm{IU}$; $\alpha$-tocopherol acetate $=$ $2.6 \mathrm{mg}$; pyridoxine hydrochloride $=2.6 \mathrm{mg}$; thiamin hydrochloride $=2.6 \mathrm{mg}$; riboflavin sodium phosphate $=1.95 \mathrm{mg}$; nicotinamide $=13 \mathrm{mg}$; ascorbic acid $=65 \mathrm{mg}$; dexpanthenol $=5.2 \mathrm{mg}$ and finely powdered sucrose to make $6.5 \mathrm{~g}$.

${ }^{5}$ Mineral mixtures (grams per kilogram dry weight of diet): $\mathrm{MgSO}_{4} \cdot 7 \mathrm{H}_{2} \mathrm{O}=$ 1.85; $\mathrm{ZnSO}_{4} .7 \mathrm{H}_{2} \mathrm{O}=0.50 ; \mathrm{MnSO}_{4} .4 \mathrm{H}_{2} \mathrm{O}=0.15 ; \mathrm{CuSO}_{4} .5 \mathrm{H}_{2} \mathrm{O}=0.020 ; \mathrm{KIO}_{3}$ $=0.0015 ; \mathrm{FeSO}_{4} .7 \mathrm{H}_{2} \mathrm{O}=0.200$

\subsection{Preparation of the HFD}

Amount of subcutaneous fat from mutton and animal butter were used as a source of saturated fat in the HFD. The fat was compressed in a robot and melted at $100^{\circ} \mathrm{C}$ for 15 min. The HFD was prepared by mixing the melted preparation of fat with the cholesterol, the chemical and ingredient compounds listed in table 1 in a stainless blender. The homogeneous diet was transformed into a piece of cake, then dried at $45^{\circ} \mathrm{C}$ and stored at $4{ }^{\circ} \mathrm{C}$ for a short time. 


\subsection{Experimental Design}

Twenty-eight adult male Wistar rats were used in the study. At their arrival, the rats were fed BD ad libitum during a short period of adjustment (1 week). After this period, the rats were weighed and randomly assigned to four groups of seven animals with comparable body weights. They were housed individually in stainless-steel wire cages. The room temperature was maintained at $25 \pm 2{ }^{\circ} \mathrm{C}$, and lit daily from 07.00 to $19.00 \mathrm{~h}$. During the EFP (9 weeks), the group 1 continues to receive the $\mathrm{BD}+$ distilled water (BD group), the group 2 received the HFD + distilled water (HFD group), the group 3 received HFD +15 -min GTD (GTD group) and the group 4 received HFD + GTDLJ mixture (GTDLJ group). The food intake was recorded throughout the EFP and quantified by weighing the amount of food spilled and refused. Every day, before tea intake, each rat was given about $5 \mathrm{ml}$ of distilled water to prevent dehydration. The volume of 15-min GTD and GTDLJ consumed by each rat was measured daily. At the end of the EFP, the rats were weighed, and then killed by decapitation. The fasting blood was drawn in vacutainer tubes, centrifuged at 3,000 rpm for $10 \mathrm{~min}$, and then the plasma was removed for the analysis of glucose, triglycerides, total cholesterol and TAS. Another aliquot of total blood was used to determine the whole blood glutathione peroxidase activity (GPX) and blood iron. In addition, the liver was removed, weighed and rinsed with a $\mathrm{Na} \mathrm{Cl}$ solution at $0.9 \%$ for histological assays.

\subsection{Analytical Methods}

\subsubsection{Analysis of Metabolic Parameters, TAS, GPX and Blood Iron}

Plasma glucose, triglycerides and cholesterol were analyzed by enzymatic colorimetric methods: GOD-PAP, GPO-PAP and CHOD-PAP using kits supplied by Biomaghreb (Tunisia). The TAS and the GPX activity were determined by Miller et al. [11], Paglia, and Valentine [12] methods, respectively. The blood iron was determined after the incineration of samples between $480-550^{\circ} \mathrm{C}$ in a muffle (STUART) for $48 \mathrm{~h}$ to obtain white ash. After being cooled to room temperature, the ashes were recovered by $2.5 \mathrm{ml}$ concentrated $\mathrm{HCl}$, taken up to $25 \mathrm{ml}$ with ultra-pure water. Iron concentration was measured by atomic absorption spectrophotometry (Perkin-Elmer 305B) using titrisol standard solutions (Merck, Darmstadt, Germany).

\subsubsection{Histological Assays}

The liver was fixed with $30 \%$ formaldehyde, dehydrated in a graded series of ethanol solutions and embedded in paraffin. Slices of $3.5-4 \mu \mathrm{m}$ thickness was cut from the paraffin blocks with a microtome, dried before staining with hematoxylin-eosin and examined with a light microscopy. The microscopy evaluation was graded negative $(-)$ in the case of no vesicular steatosis, $( \pm)$ in the case of few micro vesicular steatosis, positive $(+)$ in several micro vesicular steatosis and $(++)$ if the liver tissues contained very important micro and macro vesicular steatosis. These scores were transformed into quantitative variables: the value 0 for $(-), 1$ for $( \pm), 2$ for $(+)$ and 3 for $(++)$.

\subsubsection{Statistical Analysis}

The results were expressed as means \pm SEM. Analysis of variance followed by Student's t-test was used to compare the groups. Statistical analysis was performed with Graph Pad Prism version 5.00 for Windows, (Graph pad software Inc., San Diego, CA). Differences were considered significant at $\mathrm{p}<0.05$.

\section{Results}

Long-term effect of 15-min GTD and GTDLJ on food intake, body weight gains and relative liver weight

The long-term effect of 15-min GTD or GTDLJ on food intake, body weight gains and relative liver weight was presented in table 2 .

Table 2. Long-term effect of 15-min GTD or GTDLJ on food intake, body weight gains and relative liver weight.

\begin{tabular}{lllll}
\hline Parameters & BD group & HFD group & GTD group & GTDLJ group \\
\hline Food intake, g/day & $20.8 \pm 0.2$ & $12.4 \pm 0.1$ & $11.3 \pm 0.2$ & $11 \pm 0.1$ \\
Tea consumption, ml/day & - & - & $23.2 \pm 0.7$ & $24.5 \pm 0.7$ \\
Water consumption, ml/day & $42.5 \pm 2$ & $42 \pm 0.9$ & 5 & 5 \\
Initial body weight, g & $219 \pm 4$ & $214.7 \pm 2$ & $234.7 \pm 6$ & $210 \pm 6$ \\
Final body weight, g & $278.7 \pm 5$ & $313.5 \pm 2$ & $276 \pm 5$ & $264.8 \pm 6$ \\
Body weight gains, g & $59.7 \pm 3.7$ & $98.8 \pm 2^{* * \mathrm{a}}$ & $41.3 \pm 3.5^{* * \mathrm{~b}}$ & $54.8 \pm 4.1^{* \mathbf{c}}$ \\
Body weight gains, \% & 27.26 & 46.01 & 17.59 & 26.1 \\
Liver weight, g & $8.2 \pm 0.23$ & $9.3 \pm 0.6$ & $9.7 \pm 0.25$ & $9.4 \pm 0.4$ \\
Relative liver weight, \% & 2.94 & 2.96 & 3.51 & 3.54 \\
\hline
\end{tabular}

Legend

${ }^{\mathrm{a}}$ :Body weight gain in HFD group was significantly higher than in BD group $\left({ }^{* *} \mathrm{p}<0.001\right) .{ }^{\mathbf{b}}$ : Body weight gain of GTD group was significantly lower than HFD and BD groups, $\left({ }^{* *} \mathrm{p}<0.001\right) .{ }^{\mathbf{C}}$ The body weight gain in GTDLJ group was reversed as compared to the GTD group $\left({ }^{*} \mathrm{P}<0.05\right)$.

The volumes of GTD and GTDLJ consumed daily were comparable: $23.2 \pm 0.7$ and $24.5 \pm 0.7 \mathrm{ml}$, respectively. The rats which continue to receive the $\mathrm{BD}$ consumed significantly more food $(20.8 \mathrm{~g} / \mathrm{d})$ than those of HFD, GTD or GTDLJ groups (mean $11.5 \mathrm{~g} / \mathrm{d})(\mathrm{p}<0.01)$. The initial body weights did not differ among groups $(\sim 220 \mathrm{~g})$. However, at the end of the EFP, rats of HFD group had the highest body weight gains than the other groups. The weight gains were $59.7 \pm 3.7,98.8 \pm 2,41.3 \pm 3.5$ and $54.8 \pm 4.1 \mathrm{~g}$, which represent $27.3,46,17.6$ and $26.1 \%$ in the BD, HFD, GTD 
and GTDLJ groups, respectively. The 15-min GTD significantly reduced the body weight gains by $10 \%$ when compared to BD group and $28.4 \%$ when compared to HFD group $(\mathrm{p}<0.001)$. However, the body weight gains were reversed in the GTDLJ group as compared to GTD group, reaching a similar gain than BD group. Moreover, although the liver weights were higher in all groups receiving HFD, the relative liver weights did not significantly differ among groups.

Long-term effect of 15-min GTD and GTDLJ on metabolic parameters, TAS, GPX and blood iron

The results are presented in table 3 .

Table 3. Long-term effect of 15-min GTD or GTDLJ on glucose, lipids, TAS, GPX and blood iron

\begin{tabular}{|c|c|c|c|c|}
\hline Parameters & BD group & HFD group & GTD group & GTDLJ group \\
\hline Plasma glucose, $\mathrm{mmol} / \mathrm{L}$ & $5.6 \pm 0.1$ & $5.2 \pm 0.1$ & $4.2 \pm 0.1^{*}$ & $6.5 \pm 0.2 * *$ a \\
\hline Plasma triglycerides, $\mathrm{mmol} / \mathrm{L}$ & $1.3 \pm 0.15$ & $1.4 \pm 0.1$ & $1 \pm 0.1^{*}$ & $1 \pm 0.4$ \\
\hline Plasma cholesterol, mmol/L & $1.3 \pm 0.3$ & $3.6 \pm 0.2$ & $3.5 \pm 0.1$ & $4 \pm 0.4$ \\
\hline $\mathrm{TAS}, \mathrm{mmol} / \mathrm{L}$ & $1.15 \pm 0.3$ & $1.9 \pm 0.08$ & $3.8 \pm 0.2^{* *}$ & $3.1 \pm 0.1^{* \mathrm{a}}$ \\
\hline GPx, U/L & $27 \pm 3.8$ & $44.1 \pm 1.8$ & $57.8 \pm 1.6^{* *}$ & $42.7 \pm 4 * * a$ \\
\hline Blood iron, $\mu \mathrm{g} / \mathrm{g}$ & $204 \pm 8$ & $238 \pm 9.7$ & $112 \pm 8.5^{* *}$ & $180 \pm 10.7 * * \mathrm{a}$ \\
\hline
\end{tabular}

Legend

In GTD group: plasma glucose, triglycerides and blood iron were decreased and TAS and GPx were increased as compared to the HFD or BD groups (* $<<$ $0.05 ; * * p<0.001)$. ${ }^{a}$ The addition of LJ to GTD has reversed its effect on plasma glucose, TAS, GPx and blood iron in GTDLJ group as compared to GTD group $(* \mathrm{p}<0.05 ; * * \mathrm{p}<0.001)$.

The long-term effect of 15-min GTD significantly reduced the plasma glucose and triglycerides by 25 and $23 \%$ $(\mathrm{p}<0.01$ and 0.05 , respectively). However, 15-min GTD did not significantly influence the cholesterol profile. Moreover, the 15 -min GTD significantly increased the TAS by 230 and $95.9 \%$ and the GPX activity by 114 and $31 \%$ as compared to $\mathrm{BD}$ and HFD groups, respectively $(\mathrm{p}<0.001)$. It also significantly reduced the blood iron by 45 and $53 \%$ as compared to $\mathrm{BD}$ and $\mathrm{HFD}$, respectively. In contrast, the addition of LJ to 15-min GTD had reversed the effect of 15min GTD on plasma glucose, blood iron, TAS and GPX.

Histological evaluation of liver samples

The representative histological sections of livers from each group were shown in figure 1. No vesicular steatosis was observed in the liver of the BD group with a score of 0 (Fig1.a). However, several abundant micro and macro vesicular liver steatosis were appeared in the rats of HFD group (++) with a score of 3 (Fig1.b). In rats of 15-min GTD group, we observed only a rare presence of micro or macro vesicular steatosis in the liver $( \pm)$ with a score of 1 , suggesting that the abundant liver steatosis induced by HFD was clearly attenuated after 9 weeks of 15-min GTD treatment (Fig.1c). Surprisingly, an abundant reappearance of several micro and macro vesicular liver steatosis $(++)$ with a score of 3 in GTDLJ group was observed, suggesting that the added LJ could interact with GTD and overcome its effect on liver steatosis (Fig. 1.d).

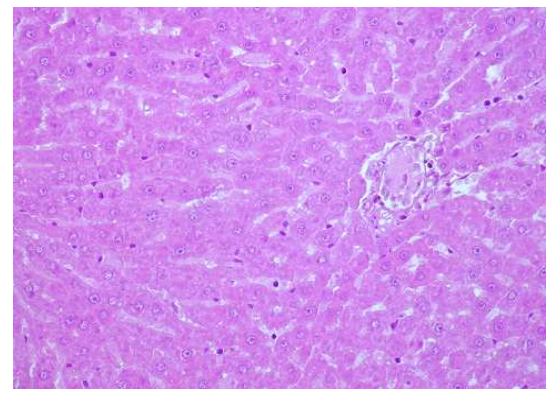

Fig. 1.a. No vesicular steatosis was noted in liver tissue of rats fed BD. CV: Central vein. (-) with a score of 0 .

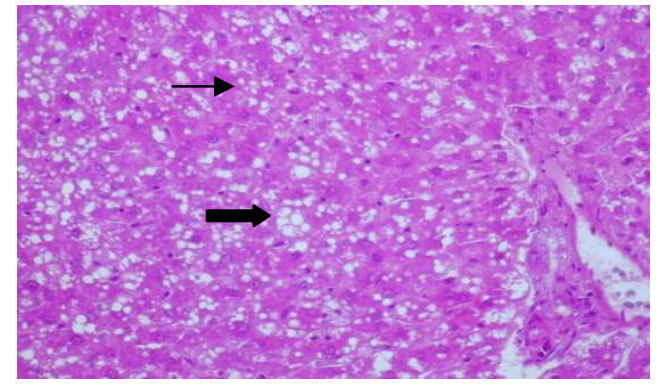

Fig. 1.b. Liver tissue of rats fed HFD + water: several micro $(\longrightarrow)$ and macro( $\longrightarrow$ ) vesicular steatosis appeared in the liver tissue. CV: Central vein. $(++)$ with a score of 3 .

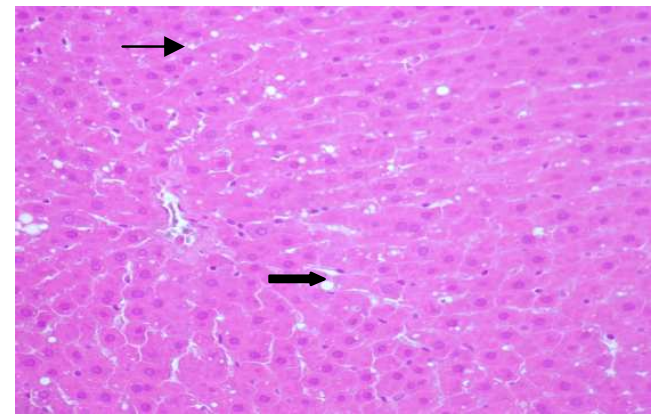

Fig. 1.c. This figure shows rarely micro $(\rightarrow)$ and macro $(\rightarrow)$ vesicular steatosis appeared in the liver tissue of rats fed HFD + GTD. CV: The central vein. ( \pm ) with a score of 1 .

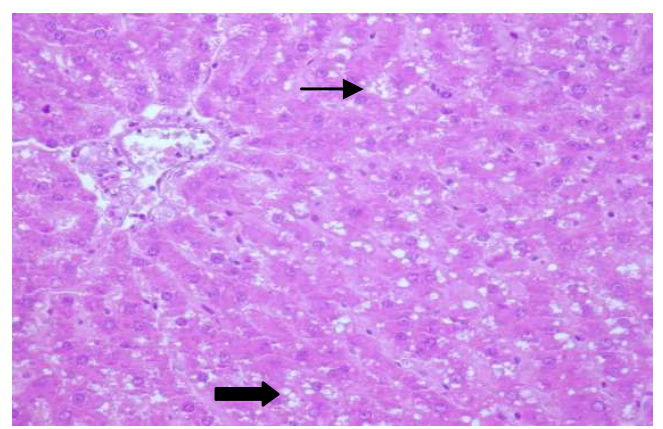

Fig. 1.d. This figure shows the reappearance of several micro $(\rightarrow)$ and macro ( $\longrightarrow$ ) vesicular steatosis in the liver tissue of rats fed HFD + GTDLJ mixture. CV: The central vein. (++) with a score of 3 . 


\section{Discussion}

Previously, we reported that cooking time of 15 -min in boiling water is appropriate to preserve high tea-polyphenol concentration and activities in GTD as compared to cooking time of 30-min, 60-min or GTI [13]. As predictable, number of compounds appeared at 15-min GTD such as epigallocatechin -3- gallate (EGCG), epicatechin-gallate (ECG) and epicatechin (EC) were reduced by about 10-times or disappeared after 30 and $60 \mathrm{~min}$ of cooking whereas some other persisted [13]. Accordingly, the 15-min GTD has the better beneficial effect on glucose absorption, glucose tolerance and strong antimicrobial activities [8, 9]. In the present study, we found that 15-min GTD significantly decreased body weight gains, HFD-induced steatosis, plasma glucose, triglycerides, and blood iron together with the improve of the antioxidant status process. This finding is in agreement with reported data about high doses of pure green tea catechins: EGCG, EGC and epicatechine that effectively reduced adipose tissue mass and ameliorated plasma lipid profiles in high-fat diet induced obese mice [14, 15]. They are also consistent with those indicating that a solution containing variable doses of EGCG or EGC or both was able to reduce body weight gain, fasting blood glucose, plasma insulin, insulin resistance and some markers of obesity-related fatty liver disease [16]. Therefore, our findings confirm that 15-min GTD has equivalent effects than those reported with purified catechins used in different experimental conditions. Consequently, cooking tea leaves in boiling water for 15-min seems did not alter tea- catechins but otherwise active polyphenols and promoted its release from tea leaves. Hence, 15-min GTD prepared under realistic Tunisian habits could constitute a good method to preserve the beneficial properties of tea-polyphenols. The beneficial effect of 15-min GTD on body weight gains, LS and related metabolic parameter could be ascribe to the modulation of lipids and glucose digestibility and absorption as well as the regulation of genes expression involved in adipogenesis, lipolysis, beta-oxidation and thermogenesis in adipose tissue [7, 17-19]. Nevertheless, the decrease of iron storage in concomitance with the reduction of body gains, liver steatosis and the improvement of the antioxidant status is very important. The cytoprotective effects of tea polyphenols against oxidative stress arise, not only from their antioxidant properties, including the scavenging of oxygen and lipid radicals, but also from their iron-chelating activity. A supplementation of the Jurkat T-cell line with green tea extract significantly decreased MDA production and DNA damage after $\mathrm{Fe}^{2+}$ oxidative treatments as compared to the control [20]. The chelating effect of tea polyphenol on iron is beneficial because the high level of stored iron is associated with an excess risk of lipid peroxidation, which is involved in the pathogenesis of liver steatosis [21]. Therefore, the depletion of iron storage by GTD could attenuate the exacerbation of liver steatosis, likely via the decrease of oxidative stress and lipogenesis in the hepatocytes [22].

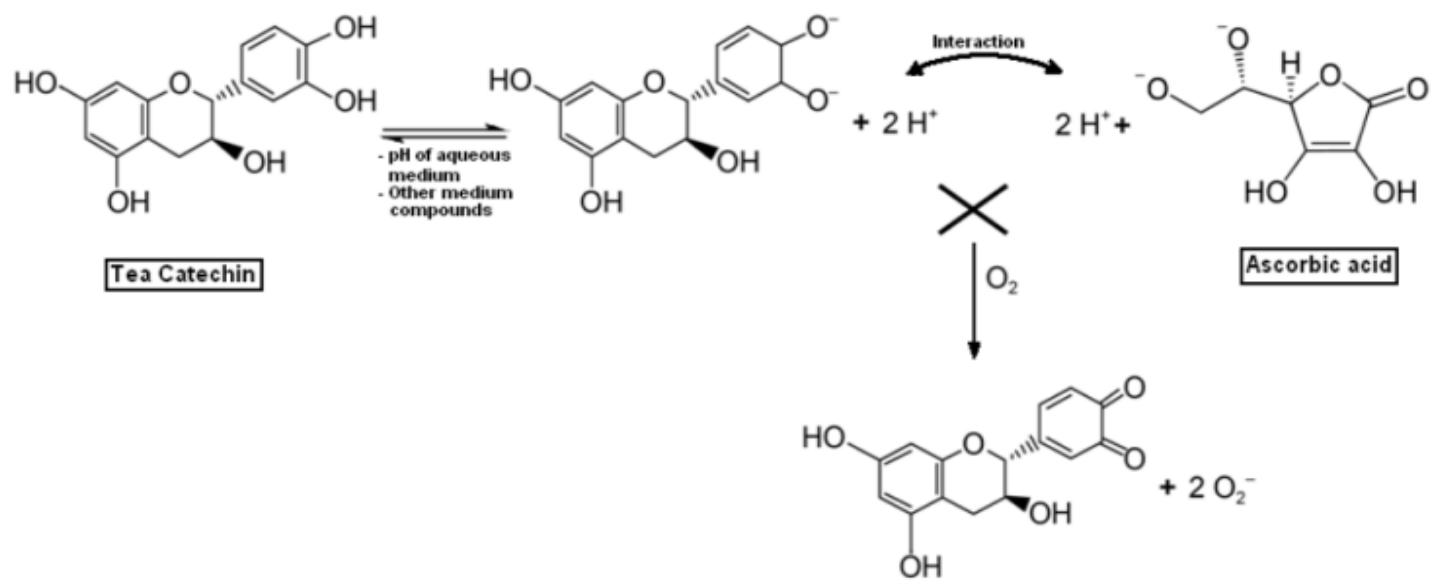

Figure 2. hypothesis of the interaction between tea catechin and ascorbic acid or other compounds from LJ.

The primary objective of our study was to investigate the long-term effect of the interaction between the $\mathrm{LJ}$ as a high source of AA and 15-min GTD. Interestingly, the addition of LJ to 15-min GTD has counteracted the effects of GTD on body weight gains, liver steatosis, plasma glucose, antioxidant markers and blood iron. Moreover, in GTDLJ group, we noticed the reappearance of several micro or macro vesicular steatosis in the liver tissue and adverse effects on body weight gains, plasma glucose, antioxidant markers and blood iron. In a similar context, we showed that the addition of graded doses of AA to 15-min GTD was able to overcome the inhibiting effect of tea decoction on iron absorption and improved its bioavailability from a typical Tunisian meal fed to rats [10]. To our knowledge, no comparable data are available because GTD is typical beverage for North Africa countries especially in Tunisia. However, a significant interaction between grape-seed polyphenols and vitamin $\mathrm{C}$ treatment was observed on blood pressure in human [23]. The vitamin $C$ alone reduced systolic blood pressure versus placebo, while polyphenols did not. However, treatment with the combination of vitamin $\mathrm{C}$ and polyphenols increased systolic and diastolic blood 
pressure in treated hypertensive individuals receiving 500 $\mathrm{mg}$ /day vitamin C, $1000 \mathrm{mg}$ /day grape-seed polyphenols for 6 weeks. Subsequently, these authors suggested caution for hypertensive subjects taking supplements containing combinations of vitamin $\mathrm{C}$ and polyphenols. In addition, it has been reported that AA offsets the inhibitory effect of bioactive dietary polyphenolic compounds on transepithelial iron transport in Caco-2 intestinal cells [24]. It is difficult to explain the interaction between GTD as a high source of catechin-polyphenols and $\mathrm{LJ}$ as a high source of AA. However, we think that the obtained data could be attributed to the active redox process reactions that may occur between the ionized hydroxyl groups of tea catechin $(5,7,3$ ', 4'tetrahydroxyflavan-3-ol) and those of AA co-present in the gastro- intestinal medium. Chemically, the bioactive properties of tea catechins, mostly arise from its ionized form which has the ability to act as free radical acceptors of donated electrons from other compounds co-present in the same medium [25]. In this case, some free radicals such as hydrogen or oxygen released from ionized molecules of AA or other LJ compounds could react with functional sites of catechins and totally or partially blocked it bioactive functions. This hypothetical mechanism could be schematized as shown in figure 2. Other data reported that AA could form covalent bonds with natural products by ascorbylation reaction. Since a number of natural products, containing an ascorbate moiety has been isolated and characterized from a variety of biological sources [26].

Therefore some ascorbylated tannins appear to be formed from electrophilic addition to dehydroascorbic acid which gives some examples of annulations of ascorbate with dietary polyphenols, e.g. EGCG and resveratrol derivatives [26]. Based on our experiment, each rat consumed $24 \mathrm{ml}$ of the LJ-GTD mixture that could theoretically provide about $2 \mathrm{mg}$ of AA to rat/day. While the amount of AA intake of rat is lower as compared to human (daily requirement: $70 \mathrm{mg}$ ), it has counteracted the effects of 15-min GTD on most of studied parameters, especially body weight gains and HFDinduced LS.

\section{Conclusion}

In summary, 9-weeks 15-min GTD treatment significantly decreased body weight gains, liver related micro and macro vesicular steatosis, plasma glucose, triglycerides and blood iron in parallel with an improvement of TAS and GPX activities. Surprisingly, the addition of LJ, as a high source of AA to GTD, has partially or totally counteract the effects of tea-polyphenols and therefore abolished its properties. Although LJ is considered as a high source of antioxidant compounds as well as GTD, there was no synergistic effect between the two kinds of antioxidant co-present in the same medium. Because GTD cooked in boiling water for a short time is beneficial, its use in the prevention of obesity and their comorbidities could be interesting to combat liver steatosis but it must be consumed without sugar or LJ because molecular interactions could abolish the beneficial effects of both tea-polyphenols and LJ.

\section{Acknowledgements}

Authors like to thank the "Ministère de l'Enseignement Supérieur et de la Recherche Scientifique en Tunisie » for supporting this work.

\section{References}

[1] Hassan K., Bhalla V., El Regal ME., A-Kader HH. Nonalcoholic fatty liver disease: A comprehensive review of a growing epidemic. World Journal of Gastroenterology. 20, 2014, pp.12082-12101. DOI 10.3748/wjg.v20.i34.

[2] Angulo P. Nonalcoholic fatty liver disease. New England Journal of Medicine. 346, 2002, pp.1221-1231. DOI 10.1056/NEJMra011775

[3] Angulo P., Lindor KD. Non-alcoholic fatty liver disease. Journal of Gastroenterology and Hepatology, 2002, 17 (Suppl.): pp. S186-S190. DOI 10.1046/j.14401746.17.s1.10.x

[4] Garg A., Misra A. Editorial: hepatic steatosis, insulin resistance, and adipose tissue disorders. Journal of Clinical Endocrinology and Metabolism, 87, 2002, pp. 3019-3022.

[5] Dowman JK., Tomlinson JW., Newsome PN. Pathogenesis of non-alcoholic fatty liver disease. The Quarterly journal of medicine, 103, 2010, pp.71-83. DOI 10.1093/qjmed/hcp158.

[6] Cabrera C., Artacho R., Giménez R. Beneficial effects of green tea- A review. The Journal of the American College of Nutrition, 25, 2006, pp.79-99. DOI 10.1186/1749-8546-5-13.

[7] Rains TM., Agarwal S., Maki KC. Antiobesity effects of green tea catechins: a mechanistic review. The Journal of Nutritional Biochemistry, 22, 2011, pp. 1-7. DOI 10.1016/j. jnutbio.2010.06.006.

[8] Snoussi C., Ducroc R., Hamdaoui MH., Dhaouadi K., Abaidi H., Cluzeaud F., Nazaret C., Le Gall M., Bado A. Green tea decoction improves glucose tolerance and reduces weight gain of rats fed normal and high-fat diet. The Journal of Nutritional Biochemistry, 25, 2014, pp. 557-564. DOI 10.1016/j.jnutbio.2014.01.006.

[9] Jlassi H. Influence du saccharose ou des édulcorants ajoutés au thé vert ou noir sur les activités antioxydantes et antibactériennes: Etude in vitro. Thesis, 2012, Faculty of Sciences of Bizerte, Tunisia.

[10] Hamdaoui M., Doghri T., Tritar B. Effect of different levels of an ascorbic acid and tea mixture on nonheme iron absorption from a typical Tunisian meal fed to healthy rats. Annals of Nutrition and Metabolism, 39, 1995, pp. 310-316. DOI: $10.1159 / 000177878$.

[11] Miller NJ., Rice-Evans C., Davis MJ., Gopina-than V., Milner A. Total antioxidant status. Clinical Sciences, 84, 1993, pp. 407-412. DOI 10.1042/cs0840407.

[12] Paglia DE., Valentine WN. Studies on the quantitative and qualitative characterization of erythrocyte glutathione peroxidase. Journal of Laboratory and Clinical Medicine, 70, 1967, pp.158-169. 
[13] Dhaouadi K. Identification et analyse structurale de certains polyphénols du thé avant et après cuisson à l'eau : Impact sur le pouvoir antioxydant et antimicrobien. Thesis, 2010, Faculty of Sciences of Bizerte, Tunisia.

[14] Chen N., Bezzina R., Hinch E., Lewandowski PA., CameronSmith D., Mathai ML., Jois M., Sinclair AJ., Begg DP., Wark JD., Weisinger HS., Weisinger RS. Green tea, black tea, and epigallocatechin modify body composition, improve glucose tolerance, and differentially alter metabolic gene expression in rats fed a high-fat diet. Nutrition Research, 29, 2009, pp 784-793. DOI 10.1016/j.lfs.2009.04.001.

[15] Uchiyama S, Taniguchi Y, Saka A, Yoshida A, Yajima H. Prevention of diet-induced obesity by dietary black tea polyphenols extract in vitro and in vivo. Nutrition, 27, 2011, pp 287-292. DOI 10.1016/j.nut.2010.01.019.

[16] Sae-Tan S., Grove KA., Kennett MJ., Lambert JD. (-)Epigallocatechin-3-gallate increases the expression of genes related to fat oxidation in the skeletal muscle of high fat-fed mice. Food Function, 2, 2011, pp.111-116. DOI $10.1039 / \mathrm{c} 0$ fo00155d.

[17] Lee MS, Kim CT., Kim Y. Green tea (-)-epigallocatechin-3gallate reduces body weight with regulation of multiple genes expression in adipose tissue of diet-induced obese mice. Annals of Nutrition and Metabolism 54, 2009, pp.151-157. DOI 10.1152/ ajpendo. 00362.

[18] Mousumi B., Joshua DL., Jihyeung J., Kenneth RR., Sue AS. The major green tea polyphenol, (-) - epigallocatechin-3gallate, inhibits obesity, metabolic syndrome, and fatty liver disease in high-fat-fed mice. The Journal of Nutrition, 138, 2008, pp.1677-1683.

[19] Park HJ., DiNatalea DA., Chunga MY., Park YK., Park JY., Kooa SI., Meeghan O'C., Jose E M., Brunoa RS. Green tea extract attenuates hepatic steatosis by decreasing adipose lipogenesis and enhancing hepatic antioxidant defenses in ob/ob mice. The Journal of Nutritional Biochemistry, 22, 2011, pp 393-400. DOI 10.1016/j.jnutbio.2010.03.009.

[20] Erba D., Riso P., Colombo A., Testolin G. Supplementation of Jurkat $\mathrm{T}$ cells with green tea extract decreases oxidative damage due to iron treatment. The Journal of Nutrition, 129, $1999,2130-2134$

[21] Silva M., Silva ME., de Paula H., Carneiro CM., Pedrosa ML. Iron overload alters glucose homeostasis, causes liver steatosis, and increases serum triacylglycerols in rats. Nutrition Research, 28, 2008, pp.391-398. DOI 10.1016/j.nutres.2008.02.009.

[22] Purohit V., Russo D., Salin M. Role of iron in alcoholic liver disease: introduction and summary of the symposium. Alcohol 30, 2003, pp 93-97. DOI: 10.1016/S0741-8329 (03)00132.

[23] Ward NC., Hodgson JM., Croft KD., Burke V., Beilin LJ., Puddey IB. The combination of vitamin $\mathrm{C}$ and grape-seed polyphenols increases blood pressure: a randomized, doubleblind, placebo-controlled trial. Journal of Hypertension, 23, 2005, pp.427-434.

[24] Kim EY., Ham SK., Bradke D., Ma Q., Han O. Ascorbic acid offsets the inhibitory effect of bioactive dietary polyphenolic compounds on transepithelial iron transport in Caco-2 intestinal cells. The Journal of Nutrition, 141, 2011, pp. 828834. DOI 10.3945/jn.110.134031.

[25] Bark KM., Yeom JE., Yang JI. Yang IJ. Park CH., Park HR. Spectroscopic studies on the oxidation of catechin in aqueous solution. Bulletin of the Korean Chemical Society, 32, 2011, pp. 3443-3447. DOI.org/10.5012/bkcs.2011.32.9.3443

[26] Kesinger NG., Stevens JF. Covalent interaction of ascorbic acid with natural products. Phytochemistry.70, 2009, pp.1930-1939. DOI 10.1016/j.phytochem.2009.09.028. 